\title{
EXPERIENCIAS FORMATIVAS DE EDUCADORES SOCIALES EN BRASIL Y PORTUGAL: REFLEXIONES COMPARTIDAS
}

\section{FORMATIVE EXPERIENCES OF SOCIAL EDUCATORS IN BRAZIL AND PORTUGAL: SHARED REFLECTIONS}

\author{
Érico Ribas Machado \\ Universidade Estadual de Ponta Grossa, Brasil \\ https://orcid.org/0000-0001-7627-4751 \\ ericormachado@gmail.com
}

Silvia Azevedo

Instituto Superior Ciencias Educativas del Duero, Portugal https://orcid.org/0000-0002-7185-6063

silvia.azevedo@iscedouro.pt

Francisco Alejandro Rincón Munevar

Universidade Estadual de Ponta Grossa. Brasil https://orcid.org/0000-0003-4938-0182 docentefran@gmail.com

Recibido: 09-12-2019 Revisado: 21-12-2019 Aceptado: 14-02-2020

Resumen: El siguiente texto presenta una reflexión sobre los caminos formativos de los Educadores Sociales en Brasil y Portugal. En el contexto portugués, los Educadores Sociales ya tienen la garantía de un curso de formación en un nivel superior. En Brasil, estos trabajadores todavía están en el proceso de exigir regulaciones específicas para su profesión, así como de garantizar su proceso de formación en un título universitario. El enfoque metodológico que orienta está reflexión es la Pedagogía Social Comparada, la cual permite la elaboración de elementos que se aproximan o distancian de realidades en torno al foco de reflexión que es la Formación de Educadores Sociales. En el caso brasileño, la formación de Educadores Sociales se ha llevado a cabo principalmente a través de proyectos de extensión vinculados a instituciones de educación superior. En el caso de Portugal, la formación se lleva a cabo en un nivel superior y los Educadores Sociales ya tienen una perspectiva profesional que los distingue de otros profesionales, un proceso construido a través de un colectivo profesional organizado.

Palabras claves: Educadores sociales en Brasil, educadores sociales en Portugal, formación profesional, educación social, pedagogía social.

\begin{abstract}
The text presents the reflection on the formative paths of social educators in Brazil and Portugal. In the Portuguese context, Social Educators already have the guarantee of a training course at a higher level. In Brazil, these workers are still in the process of claiming, demanding specific regulations for their profession, as well as guaranteeing their training process in a university career. The methodological approach that organizes this reflection is the Comparative Social Pedagogy that allows the elaboration of elements that approximate or distance realities around the focus of reflection that is the Formation of Social Educators. In the Brazilian case, the training of Social Educators has been carried out mainly through extension projects linked to Higher Education Institutions. In the case of Portugal, the training is carried out at a higher level and the social educators already They have a professional perspective that distinguishes them from other professionals, a process built through an organized professional collective.
\end{abstract}


Keywords: Social educators in Brazil, social educators in Portugal, professional education, social education, social pedagogy.

Cómo citar este artículo: Ribas Machado, É., Azevedo, S. y Ricón Munevar, Fco. A,. (2020). Experiencias formativas de educadores sociales en Brasil y Portugal: reflexiones compartidas. Hachetetepé. Revista cientifica en Educación y Comunicación, (20), 100-107.doi: http://doi.org/10.25267/Hachetetepe.2020.i20.12

\section{INTRODUCCIÓN}

Los autores de este texto compartirán con ustedes, lectores, dos relatos de experiencias que cuentan historias cómo ocurren algunas cuestiones formativas y de trabajo en el caso de los Educadores Sociales en Brasil y Portugal. Las reflexiones presentadas están inspiradas en la Educación Comparada (García Garrido, 1986), que permitió la creación de un enfoque metodológico denominado Pedagogía Social Comparada (Ribas Machado, 2014). Esta perspectiva permitió a los tres autores del texto compartir información que se refiere al intercambio de cartas entre los autores que están en Brasil, Érico y Alejandro y el autor que está en Portugal, Silvia, para compartir aspectos históricos de experiencias también formativas en sus respectivos territorios de análisis. Las acciones intercambiadas permiten relacionar elementos que establecen distancias y aproximaciones entre las realidades, lo que genera el aspecto comparativo de la propuesta de este texto inspirado en un formato de carta.

\section{Brasil, febrero de 2020}

\section{REALIDAD DE LOS EDUCADORES SOCIALES BRASILEÑOS}

Compartimos con ustedes nuestras conversaciones sobre la investigación que se está llevando a cabo en el contexto brasilero entorno al origen, proceso de formación y regulación para la profesionalización de los Educadores Sociales. Podemos identificar a través del estudio realizado por Ribas Machado (2014), que históricamente la figura del Educador Social comienza a aparecer en el escenario educativo brasileño a finales de la década de 1990 e inicio de los años en 2000. Son trabajadores oriundos de movimientos sociales, principalmente de movimientos en defensa de los derechos de los niños y adolescentes.

Mediante la lectura de documentos y publicaciones de investigación de maestría (Dias, 2018) y doctorado (Bauli, 2018) conseguimos acceder a informaciones y reflexiones que apuntan sobre los indicios de cómo los Educadores Sociales pasan a componer el cuadro de profesionales que trabajan específicamente en las políticas de Asistencia Social en el Brasil. Son trabajadores reconocidos en el Código de Ocupaciones de Brasil (CBO) y están admitidos en los niveles de enseñanza media o hasta enseñanza fundamental para trabajar en las políticas antes mencionadas.

En la década de 1990 vivenciamos en nuestro amado Brasil, hasta entonces, el apogeo del neoliberalismo ordenado para América Latina, exacerbado en Chile. Fue bajo el gobierno de Fernando Henrique Cardoso (1995-2002) que experimentamos la reforma del Estado brasilero que permitió una consolidación aún más profunda de la pobreza generalizada y la riqueza concentrada. Con la mayoría pobre y hambrienta, el nuevo presidente llamado Luiz Inácio Lula da Silva (2003-2010) fue elegido a principios de la década del 2000, lo que inspiró esperanza y la posibilidad de construir un Brasil menos desigual. Contrariamente a los discursos equivocados y falsos, Lula y

e-ISSN:2172-7910

Doi: http://doi.org/10.25267/Hachetetepe.2020.i20.12

Universidad de Cádiz 
su sucesora, la presidenta Dilma Roussef (2011-2016), implementaron una política postneoliberalismo (Sader y Gentilli), lo que significa un nuevo modelo de gestión del Estado dentro del capitalismo, que no tiene nada que ver con el comunismo, falsamente promocionado.

Optamos por contextualizar de manera breve el recorrido de los gobiernos brasileños más recientes, para poder localizar la actuación de Educadores Sociales como un reflejo de esas influencias. Este reflejo es presentado por Ribas Machado (2010) en su disertación.

Elegimos contextualizar brevemente el camino de los gobiernos brasileros recientes para ubicar el trabajo de los Educadores Sociales como reflejo de estas influencias. En la década de 1990, en el pico neoliberal, se alentó a los movimientos sociales a transformarse en organizaciones no gubernamentales, ya que la lógica del Tercer Sector llega con toda su fuerza a Brasil, lo que inspira la responsabilidad de la sociedad civil de garantizar elementos competitivos, a priori, al Estado. Este proceso resultó en un cambio en la relación entre el Estado y la Sociedad Civil, ya que hasta entonces los Movimientos Sociales se fortalecieron en Brasil, principalmente con la influencia del proceso de apertura democrática, rompiendo con la Dictadura y la elaboración de la Constitución Federal de 1988, la población se sintió responsable de acusar al Estado por su papel en la garantía de sus derechos. Con la brisa de la tercera ola, esta relación se modifica, ya que la población comienza a sentirse responsable de garantizar estos derechos junto con el Estado o incluso en el lugar del Estado. La historia muestra que un estado debilitado, la pobreza y la desigualdad social se ven agravados, y se necesitan más educadores para trabajar con grupos humanos. De esta manera, los exmilitantes de los movimientos sociales comenzaron a actuar como activistas de derechos humanos, pero a través de las ONG, un contexto que se hizo más complejo a lo largo de los años.

El Educador Social, quien es visto como un trabajador en las políticas de Asistencia Social en Brasil, es el resultado, por lo tanto, de toda la historia previa. Originado en la militancia en los movimientos sociales, pasó por la lucha por los derechos humanos en las ONG y en las últimas dos décadas es un profesional contratado a través de concursos o procesos de selección temporal en municipios y estados brasileños. No podemos dejar de relatar y explicar que estamos dirigiendo este intercambio de historias para hablar más específicamente sobre el Educador Social reconocido en una política estatal, pero sabemos que, dado que no existe una regulación de esta profesión en Brasil, encontramos Educadores Sociales con diferentes nomenclaturas y terminologías, actuando en varios espacios además de las políticas estatales. Es esencial explicar que el establecimiento de políticas públicas más consistentes en el ámbito de la Asistencia Social no significó el fortalecimiento del Estado frente a la sociedad civil organizada, por el contrario. El Estado pudo regular y fortalecer la asociación con el Tercer Sector, es decir, el Educador Social está muy presente, es decir, en prácticamente todas las instituciones no gubernamentales que prestan servicios públicos.

Esta historia que compartimos nos inspirará en nuestra defensa del proceso de capacitación de los Educadores Sociales y cuál debería ser el estatus profesional de este trabajo. En un análisis comparativo con docentes que actualmente se encuentran en las escuelas brasileñas y reguladas en la Ley de Directrices nacionales de Educación - LDB 1996, la exigencia de formación en cursos de posgrado en Educación Superior, debido a 
que es reconocida la complejidad y la importancia de los procesos educativos que ocurren en las escuelas. Nos preguntamos cómo son los procesos educativos que ocurren en otras instituciones educativas diferentes a las escuelas, ¿Cuál es la justificativa para que cualquier funcionario con enseñanza media o fundamental pueda actuar como Educador Social? Este tema que nos instiga a un intenso debate con diferentes grupos en diferentes espacios además de Brasil, no menosprecia a los demás profesionales que solo tiene este nivel de educación. Pero confirma la necesidad de pensar en la formación de Educadores Sociales que actuaran con niños, adolescentes, jóvenes, adultos y adultos mayores, con sus diversas demandas y no el caso brasileño, por supuesto, solo demandas relacionadas con problemas sociales, económicos y psicológicos.

Para su conocimiento, relatamos que conocemos los cursos de extensión para Educadores Sociales en las siguientes universidades brasileras: Universidad Estatal de Ponta Grossa (UEPG), Universidad Estatal de Maringá (UEM), Universidad Federal de Paraná (UFPR), Universidad Federal de Río Grande del Sur (UFRGS), Universidad Federal de Paraíba (UFPB); Universidad Estatal de Bahía (UNEB); Universidad de San Pablo (USP); Universidad Estatal de Río de Janeiro (UERJ); Universidad Federal de Espirito Santo (UFES).

Para finalizar este intercambio, pero sin terminar nuestra conversación, es importante decir que el siguiente paso significa la producción investigaciones cada vez más direccionadas a analizar, registrar y categorizar estas experiencias de formativas, contribuyendo oficial y directamente para la elaboración del mencionado currículo.

\section{Portugal, febrero de 2020}

\section{REALIDAD DE LOS EDUCADORES SOCIALES PORTUGUESES}

Bien iniciando nuestra conversación sobre la realidad de los Educadores Sociales portugueses, cabe mencionar para iniciar la historia de la profesión. Sabían que la Educación Social es una profesión relativamente reciente (42-45 años) en Portugal (Azevedo y Correia, 2013). Todavía estamos en el proceso de profesionalizar y de búsqueda de nuestra identidad. Ya sea por nuestra versatilidad, carácter innovador y emancipatorio, Es una profesión de complejidad. En primer lugar, debido a los procesos inherentes a la evolución de la profesión, con respecto a la forma en que la Educación Social se ha desarrollado en cuanto formación en nivel superior, especializada y académica, asumiendo nuevas configuraciones (Pérez Serrano, 2003). En un corto período de tiempo, los educadores sociales portugueses dejaron de estar apenas asociados con una perspectiva técnica, sino que también asumen un papel destacado en la investigación e intervención psicosocial. La complejidad, el rigor y la responsabilidad del trabajo realizado por el educador social exigen una formación de nivel superior, saberes y posturas para adaptarse a los nuevos desafíos y realidades de la sociedad actual. No es posible educar sin el conocimiento de la epistemología y la metodología del campo educativo. La Educación Social se adaptó a los cambios sociales, reconfigurándose social y profesionalmente, su reconocimiento profesional se logró a través de sus prácticas, a través de sus proyectos sociopedagógicos, que van demostrando la relevancia de nuestra profesión. La Educación Social en Portugal abarca sus propios principios éticos y deontológicos y su matriz de referencia es la Pedagogía Social Alemana, una variante de las Ciencias de la Educación. 
El desarrollo y expansión de la Educación Social, en Portugal, se convierte en una multiplicidad de factores, comunes como modelos de Educación Social españoles y francófonos. La complejidad creciente del mundo, los cambios constantes en el desarrollo tecnológico han llevado a cambios en nuestras formas de vida y formas de relacionarnos con las personas. Quien no concuerda, vive corriendo y muere corriendo, sin duda hay en todo el mundo, desencadenado por el capitalismo desenfrenado, un aumento de los desfavorecidos, la marginación y la exclusión social que resultan del desempleo y la inseguridad laboral, las sanciones profesionales y sociales, especialmente de los grupos más debilitados. Todo esto, impuso al Estado una evaluación del papel político-social, de los trabajadores e interlocutores sociales y del tipo de intervención desarrollada como asistencialista. Desde 1980, en Portugal, las políticas sociales se han observado "ya sea en (...) su conceptualización y particularidades, o en la exploración de varias áreas y sectores específicos de su materialización (salud, educación, vivienda, seguridad social, etc." (Rodrigues, 1999: $18)$.

Es precisamente en este momento de intervención asistencial, que se produce la relación e interacción entre el Servicio Social y la Pedagogía / Educación Social. Y sepan que durante este tiempo el Estado buscó nuevas formas de intervención basadas en políticas socioeducativas, con entornos favorables para el desarrollo de las personas y la automatización. De hecho, el fracaso de las políticas sociales ha llevado a un aumento de la privación de los derechos de la ciudadanía. La Educación Social surge de la comprensión de que el trabajo social requiere nuevas políticas de intervención social de una manera más pedagógica y menos social, ya que la asistencia o asistencia se convertirá en una necesidad reductora (y contradictoria) de intervención social. Todo esto sucede en un panorama de progresiva valorización de los Derechos Humanos, donde la Declaración Universal de los Derechos Humanos en Portugal fue decisivo para el fortalecimiento de la Educación Social, promoviendo los intereses de las ideas humanitarias de igualdad social, justicia, solidaridad, fraternidad y hospitalidad (Azevedo y Correia, 2013).

Otro factor decisivo para el surgimiento de la Educación Social en Portugal fue un cambio en el concepto de educación: la educación deja de ser un activo particular de la escuela y comienza a tener nuevas dimensiones: la dimensión socioeducativa. Además de la educación formal, se consideró la educación no formal e informal, es decir, una educación con una función integral permanente de diferentes contextos de emergencia, de paradigmas, como el aprendizaje permanente, dedicado a teorías y modelos de educación y formación, de la pedagogía social, que valora el aprendizaje de la experiencia de la vida. Educar para el progreso humano implica personas, pretensiones, necesidades y proyectos de vida futura (Delors, 2003).

Para hacerle saber toda nuestra historia aquí en Portugal, fue realmente en la década años 70 a 80 que se marcó el comienzo y el desarrollo de la formación y la investigación en ciencias de la educación. También fue en este momento que nació el curso de educación, inicialmente como un curso técnico profesional (nivel III), equivalente al grado $12^{\circ}$ de escolaridad, luego como una licenciatura, con una duración de tres años, nivel II. Y, finalmente, después de reconocer la necesidad de formar más profundamente a los Educadores Sociales y de proporcionarles metodologías e instrumentos de intervención, surge el primer título en Educación Social de Nivel I, aprobado en 1996, en una universidad de la ciudad de Porto (Azevedo, 2011). 
Este hito fue decisivo para que la Educación Social en nuestro país. Hasta la fecha el educador social era entendido como un simple ejecutor de actividades recreativas, donde no se realizaba ningún ejercicio de la función de toma de decisiones en la intervención social, esta evolución permite que los educadores lleguen a ser vistos como técnicos superiores especializados, con cientificidad y con el conocimiento teórico necesario para desarrollar la intervención psicosocial y el trabajo de investigación y no solo como animadores sociales culturales. La formación superior permitió una mejor preparación de los profesionales, principalmente a través de las pasantías curriculares que facilitan la articulación teórica y práctica.

Permítanme decirles que a pesar de que no existen formaciones de nivel II y III en Portugal, debido al Tratado de Bolonia que trajo una cierta estandarización en la capacitación de educadores sociales en Portugal, es importante agregar que todavía hay profesionales de la educación social para desarrollar en instituciones este tipo de niveles. Sin embargo, además de la coexistencia de tres niveles de formación en educación social en un corto período de tiempo (con diferentes objetivos, grados de formación y niveles de rendimiento, durante varios años, entre la formación de nivel I, diferentes designaciones de educación social, como la Educación Social Gerontológica, la Educación Sociocomunitaria o la Educación Socioprofesional. Hoy eso ha cambiado $\mathrm{y}$ todos los cursos de educación superior tienen el mismo nombre: Educación Social, ni pueden imaginar la importancia que tuvo este cambio en la identidad profesional (Azevedo, 2011).

La identidad profesional de los educadores sociales también hizo difícil abordar el trabajo social e intervenir en el mismo contexto. Los educadores sociales actúan en contextos sociales y, por esta razón, también son trabajadores sociales. Por esta razón, el enfoque de otros profesionales sociales, como los trabajadores sociales, es en la medida que se refieren a las similitudes en la intervención, no solo cerca de los contextos y a los destinatarios. Sin embargo, el trabajo de los educadores sociales en Portugal es concebido a partir de una perspectiva educativa, transformadora y emancipadora, que supera las lógicas de asistencialismo y se basa en la centralización de las personas, en su capacitación y en su desarrollo. Los educadores sociales pretenden que las personas con los cuales trabajan puedan protagonizar sus procesos de vida. $\mathrm{O}$ el modelo de educación social portugués privilegia, la participar como eje central del trabajo socioeducativo.

Aquí la educación social se integra cada vez más en el área del trabajo social, involucrando una gama de especialidades y campos de intervención. La interdisciplinariedad del trabajo social es evidente, donde en equipo y con profesionales de diferentes áreas, se viabilizan planos, programas, proyectos de implementación, monitoreo y evaluación en relación al tejido social y la educación no formal. Aquí el Técnico Superior de Educación Social alcanza un papel notable, utilizando diversas áreas y metodologías: animación sociocultural, acciones socioeducativas, acciones comunitarias, educación de adultos, educación gerontológica, educación para la prevención, minimización y reducción de riesgos y educación personalizada $\mathrm{y}$ comunitaria (Baena, Sáenz y Quintaba-Cabañas, 2002; Petrus, 1997).

\section{CONSIDERACIONES FINALES}

La perspectiva de la Pedagogía Social Comparada nos lleva justamente a la posibilidad de presentar informaciones y reflexiones que tienen peculiaridades específicas basadas en la historia o la experiencia de cada ubicación. En este texto 
percibimos el proceso de comprensión por parte de los autores sobre nuestros contextos de actuación como investigadores. El intercambio de relatos, inspirados en la escritura de una carta, se vuelve significativo para establecer elementos comparativos. Las etapas de desarrollo del campo de la Educación Social en Brasil y Portugal son diferentes, el momento de discusión del trabajo y la formación de los Educadores Sociales en Portugal muestra el camino aun por recorrer de Brasil. Pero las experiencias brasileñas también muestran diferencias en la trayectoria, resumiendo los enfoques y distancias entre las realidades narradas. Incluso con las peculiaridades de Brasil y Portugal, la investigación de la Pedagogía Social Comparada nos instiga, precisamente a pensar la cuestión de percibir, aunque diferentes, que existe un campo de Pedagogía Social común en los dos territorios.

Terminamos, con todo, con una certeza que en Portugal, la pertinencia de la Educación Social se acentúa cada vez más, lo que se fundamenta por la formación de nivel superior, con el aumento del número de instituciones de enseñanza que apuestan en los diplomados en Educación Social. En verdad, los educadores sociales han sido integrados en nuevas políticas sociales y educativas, nuevos contextos de intervención y nuevas funciones. Las materias que definen la educación social para nosotros son de carácter interdisciplinar, (inter)cultural e (inter)estructural y exigen que se reúna diferentes estrategias y métodos de intervención de la pedagogía y de la educación social.

Entre tanto, terminamos con una certeza, en Portugal, la relevancia de la Educación Social se acentúa cada vez más, lo que se basa en la educación superior, con el aumento en el número de instituciones educativas que apuestan por los grados de Educación Social. De hecho, los educadores sociales se han integrado en nuevas políticas sociales y educativas, nuevos contextos de intervención y nuevas funciones. Los temas que definen la educación social para nosotros son de naturaleza interdisciplinaria, intercultural e interestructural y requieren que se combinen diferentes estrategias y métodos de intervención de pedagogía y educación social.

El papel del Educador Social en Portugal se ha construido a lo largos de los tiempos. La educación social es actualmente una profesión que ha madurado, en la cual, se elogia cada vez más el papel del educador social, por lo que contribuyó mucho a la praxis de los propios profesionales, lo cotidiano de su labor muestra la pertinencia y relevancia de su papel, exploraron nuevos espacios de afirmación de identidad. Es en el escenario que los profesionales de la educación social van acompañando los procesos de reconfiguración de la profesión. Asumiéndose como una educación social que se presenta como transformadora de realidades sociales, deberá por tanto tener las cualidades transformadoras, capaz de adaptarse a las múltiples reconfiguraciones sociales (Timóteo, 2013).

Hoy en día, los educadores sociales son una profesión con relevancia social debido a las funciones que desempeñan, específicamente en Portugal. Poseen un conocimiento autónomo con un universo de referencia antropológico, epistemológico y axiológico para toda su práctica profesional.

\section{REFERENCIAS BIBLIOGRÁFICAS}

Aptses, Associação dos Profissionais Técnicos Superiores de Educação Social. (2018). Revista Praxis Educare. Porto, Portugal, DF. 
Azevedo, S. (2011) Técnicos Superiores de Educação Social. Necessidade e pertinência de um estatuto profissional. Porto: Fronteira do Caos.

Azevedo, S. y Correia, F. (2013). A Educação Social em Portugal: evolução da identidade profissional. Revísta de Educación Social (RES), 17, 1-11.

Baena, M., Sáenz, J. y Quintana Cabañas, J. (2002) Pedagogía Social. Madrid: Pub. UNED.

Bauli, R. A. (2018). Educador social no brasil: profissionalização e normatização. Tese (Doutorado em Educação) - Universidad Estadual de Maringá. Maringá.

Bourdieu, P. y Passeron, J. (2009). A Reprodução: A Reprodução: Elementos para uma Teoria do Sistema de Ensino. Lisboa: Ed. Vega.

Brasil (1996). Lei de Diretrizes e Bases da Educação Nacional. Lei número 9394, 20 de dezembro de 1996.

Carneiro, R. (2001). Fundamentos da Educação e da Aprendizagem. Vila Nova de Gaia: Fundação Manuel Leão.

Delours, J. (1998). Educação um tesouro a descobrir - Relatório para a UNESCO da Comissão Internacional sobre Educação para o século XXI. Lisboa: Edições ASA.

Dias, S. P. (2018). Educadoras e educadores sociais de Porto Alegre em busca de reconhecimento. Dissertação (Mestrado) - Pontifícia Universidade Católica do Rio Grande do Sul. Programa de Pós-Graduação em Ciências Sociais. Porto Alegre.

Freire, P. (1967). Pedagogia do Oprimido. Rio de Janeiro: Paz e Terra.

García Garrido, J. L. (1986). Fundamentos de Educación Comparada. Madrid: Dickinson.

Pérez-Serrano, G. (2003). Pedagogía Social - Educación Social. Construcción científica e intervención práctica. Madrid: Narcea.

Ribas Machado, É. (2010). A constituição da Pedagogia Social na realidade educacional brasileira. Dissertação (Mestrado) - Universidade Federal de Santa Catarina. Programa de Pós-Graduação em Educação. Florianópolis.

Ribas Machado, É. (2014). O desenvolvimento da Pedagogia Social sob a perspectiva comparada: o estágio atual no Brasil e Espanha. Tese (Doutorado em Educação) - Universidad de São Paulo: São Paulo.

Rodrigues, F. (1999). Assistência Social e Politicas Sociais em Portugal. Lisboa: ISSScoop -Departamento Editorial.

Sader, E. y Gentilli, P. (Orgs). (2008). Pós-neoliberalismo: as políticas sociais e o Estado democrático: As políticas sociais e o Estado democrático. Paz e Terra: São Paulo. 